\title{
Utility of bronchoscopic intra-tumoral alcohol injection to restore airway patency
}

\author{
Michael A. Jantz ${ }^{1 \#}$, Mohamed Omballi" ${ }^{2 \#}$, Bashar N. Alzghoul' ${ }^{2}$, Sebastian Fernandez Bussy ${ }^{3}$, \\ David Becnel $^{4}$, Adnan Majid ${ }^{5}$, Hiren J. Mehta ${ }^{2}$
}

${ }^{1}$ Pulmonary Medicine, North Florida Regional Medical Center, Gainesville, FL, USA; ${ }^{2}$ Division of Pulmonary, Critical Care and Sleep Medicine, University of Florida, Gainesville, FL, USA; ${ }^{3}$ Division of Pulmonary, Critical Care and Sleep Medicine, Mayo Clinic, Jacksonville, FL, USA; ${ }^{4}$ Division of Pulmonary, Critical Care and Sleep Medicine, Tulane University, New Orleans, LA, USA; ${ }^{5}$ Division of Pulmonary and Critical Care Medicine, Beth Israel Deaconess medical center, Boston, MA, USA

Contributions: (I) Conception and design: MA Jantz, BN Alzghoul, HJ Mehta; (II) Administrative support: MA Jantz, M Omballi, BN Alzghoul, S Fernandez Bussy, D Becnel, A Majid, HJ Mehta; (III) Provision of study materials or patients: MA Jantz, HJ Mehta; (IV) Collection and assembly of data: M Omballi, BN Alzghoul; (V) Data analysis and interpretation: BN Alzghoul; (VI) Manuscript writing: All authors; (VII) Final approval of manuscript: All authors.

"These authors contributed equally to this work.

Correspondence to: Hiren J. Mehta, MD, FCCP, Director of Interventional Pulmonology. Division of Pulmonary, Critical Care and Sleep Medicine, University of Florida 1600 SW Archer Road-Room, M452 Box 100225 Gainesville, FL 32610-0225, USA. Email: hiren.mehta@medicine.ufl.edu.

Background: In cases of malignant airway obstruction, achieving airway patency using conventional bronchoscopic modalities can be challenging and may not be satisfactory. We aim to present our experience of using intra-tumoral alcohol injection (ITAI) to help achieve airway patency in malignant airway obstruction.

Methods: A retrospective study of adult patients presenting with malignant airway obstruction who underwent bronchoscopy with ITAI at a university center between 2015 and 2020 was conducted. We present procedural and patients' characteristics along with any additional bronchoscopic or systemic interventions received. Patients were classified based on airway patency response (assessed qualitatively by an interventional pulmonologist) into those with good response ( $\geq 50 \%$ improvement in airway patency) vs. those with low response ( $<50 \%$ improvement in airway patency).

Results: Forty-two patients underwent ITAI, of which 34 (81\%) had at least two bronchoscopies to evaluate response. The patient median age was 65.5 [interquartile range (IQR) 57.8-72]. Most patients had stage IV malignancy at the time of the bronchoscopy (57.1\%) and had Eastern Cooperative Oncology Group (ECOG) performance status score of between 2-3. Eighty-two percent of the patients achieved good airway patency response at the conclusion of the procedures. The procedures were generally well-tolerated and only one patient had significant bleeding that was not directly related to ITAI and required intensive care unit monitoring for 24 hours after the procedure.

Conclusions: Dehydrated alcohol is readily available, inexpensive and a safe drug that can be potentially injected directly in malignant airway obstructive lesions in a multimodality approach to achieve airway patency when traditional modalities reach their limitation. Further studies are warranted to determine whether ITAI is superior to other interventional methods, explore its utilization to treat peripheral malignant tumors, as well as to standardize the treatment protocol and determine the effects of ITAI on patient symptoms and quality of life.

Keywords: Bronchoscopy; malignant; airway obstruction; lung cancer; alcohol; tumor debulking

Submitted Apr 27, 2021. Accepted for publication Jul 23, 2021.

doi: $10.21037 /$ jtd-20-3554

View this article at: https://dx.doi.org/10.21037/jtd-20-3554 


\section{Introduction}

Malignant airway obstruction complicates one third of primary lung cancers and can develop in other malignancies metastatic to the thorax either on the initial presentation or subsequently arising later in the disease process (1). It can cause a significant burden of symptoms including cough, dyspnea, hemoptysis, and can be complicated by sepsis secondary to post-obstructive pneumonia which can eventually lead to death if untreated (2). When dealing with malignant airway obstruction, one treatment goal is to establish a patent airway, while allowing for additional systemic cancer therapies to be administered, hence improving symptoms, functional status, quality of life and potentially survival rate.

Systemic therapies for lung cancer such as radiation and chemotherapy can be effective in treating central airway obstruction but can take several days to weeks to achieve effective tumor shrinkage (3-6). Local bronchoscopic interventions can rapidly palliate symptoms, but the effects are usually short lived (6). Rapid results are usually achieved using different bronchoscopic modalities of debulking which can be challenging in some cases with prolonged procedures durations $(1,7)$.

Alcohol embolization has been used to treat nonresectable hepatocellular carcinomas (HCC) since the early 1980 s $(8,9)$. It has also been used to treat renal tumors (10) and arteriovenous malformations (11). Ethyl alcohol has been extensively used as a successful sclerosing agent for interventional therapy of many disorders, including malignancies such as intraabdominal tumors and endocrine system tumors $(10,12-15)$. It is easily accessible, biocompatible, and relatively non-toxic if carefully used (14).

The authors at the University of Florida (UF) have been using intra-tumoral alcohol injection (ITAI) to help relieve malignant central airway obstruction and reduce the duration of the debulking procedures. Our rationale to use the medication bronchoscopically was based on prior experience and studies in renal and HCC and although direct comparisons cannot be made between sites, we based our use on the fact that it is been safely injected intratumorally in the past. Dose of $3-5 \mathrm{mls}$ was chosen based on experience from prior studies for ablation of HCC and lower end of spectrum was chosen. In this study, we sought to present our experience with this novel modality.

We present the following article in accordance with the STROBE reporting checklist (available at https://dx.doi. org/10.21037/jtd-20-3554).

\section{Methods}

We conducted a retrospective cohort study of adult patients presenting with malignant airway obstruction who underwent bronchoscopy with ITAI at the UF Health System, Gainesville, Florida between April 2015 and January 2020. The study was conducted in accordance with the Declaration of Helsinki (as revised in 2013). We received approval for the study from the UF Institutional Review Board (protocol no. IRB202001086). All patients signed informed consent before the procedure.

\section{Study objectives}

The primary objective of this study was to describe the efficacy of ITAI when used with other local bronchoscopic interventions to achieve airway patency. A secondary objective of the study was to report the safety of ITAI and any secondary complications.

\section{Procedure description}

All procedures were performed by the interventional pulmonologists $(n=2)$ at our institute in the bronchoscopy suite with an interventional pulmonary fellow in training. Each case was done independently by one interventional pulmonologist. All procedures were performed under moderate sedation in spontaneously breathing patients using a T180 flexible bronchoscope (Olympus) with a $3 \mathrm{~mm}$ working channel. Medications used for sedation included fentanyl [125 mcg, interquartile range (IQR) 100-150] or meperidine (75 mg, IQR 50-75) plus midazolam (5 mg, IQR 4-7); propofol was used in one patient. We used $98 \%$ dehydrated alcohol, at a dose of 3-5 mL per patient. ITAI was considered by the interventional pulmonologist during the initial therapeutic bronchoscopy for tumor debulking if conventional bronchoscopy and ablation modalities [i.e., argon plasma coagulation (APC), forceps debulking, and/or CoreCath ${ }^{\mathrm{TM}} 2.7 \mathrm{~S}$ electrosurgical device] did not achieve airway patency either due to disease being too bulky or due to extensive mucosal infiltration or if bleeding from debulking was too extensive and precluded further tumor debulking. All patients who got an initial dose of ITAI underwent a follow up bronchoscopy for further debulking and clearance of airway slough in 2-7 days after initial bronchoscopy unless patient and family decided to pursue comfort measures only and not to have any further interventions. The degree of bronchial narrowing was 
visually estimated at the beginning of the first procedure and at the conclusion of the last procedure. The achieved airway patency was qualitatively calculated as percent in reduction in airway obstruction [(original obstructionresidual obstruction)/original obstruction $\times 100 \%$ ] at the end of the final bronchoscopy as assessed visually. We classified patients based on response into those with good response $(\geq 50 \%)$ and those with low response $(<50 \%)$. We were unable to classify patients who did not have repeat bronchoscopy. ITAI was administered in addition to other therapies as indicated. All patients signed informed consent before the procedure.

The target tumor was visualized bronchoscopically and ITAI was injected using a flexible 19/21/22-gauge Wang needle as per the bronchoscopists' discretion. The alcohol volume and number of passes were based on the bronchoscopist's discretion and the size of the tumor. All patients had one or at most two injections intratumorally. The volume ranged from $3-5 \mathrm{~mL}$ (total, regardless of number of injections performed) injected with fanning technique to disperse the alcohol throughout the lesion. Some patients required subsequent injections in repeat bronchoscopies.

The bronchoscopist decided whether to use APC, balloon dilation, mechanical debulking, and/or endobronchial stent placement based on the anatomic variation and the nature of the obstruction. We used APC to help with bleeding control and as ablative therapy using 40 watts of pulse energy and pulse duration set at 0.6 second. We also used forceps and the bronchoscope for mechanical debulking as well as an electrocautery snare for tumor resection in some cases. ITAI was used at the end of the debulking procedures. We never used APC post ITAI during the same procedure to avoid risk of airway fire.

\section{Data abstraction and analysis}

We manually reviewed patients' charts and collected data about demographics, tumor histopathology, cancer stage, tumor location, technical interventional pulmonary procedural details, concurrent systemic therapy, Eastern Cooperative Oncology Group (ECOG) performance status and survival data. Estimation of the degree of obstruction were made by a single interventional pulmonologist. All patients had a $\geq 80 \%$ endobronchial occlusion at the beginning of the first procedure.

We summarized the distribution of baseline characteristics and outcomes as percentages for categorical variables and medians with IQR for continuous variables. We compared between patients with good response and those with low response. We used the Mann-Whitney U test for continuous variables and Chi-square or Fisher's exact test as appropriate for categorical variables. All data analyses were done using IBM SPSS Statistics for Windows, Version 23.0 (released 2015, IBM Corp., Armonk, NY, USA). We elaborated further about the details of selected cases and provided selected bronchoscopic pictures.

\section{Results}

\section{Clinical characteristics}

Our initial query of the interventional bronchoscopy cases in the study period included 245 tumor debulking cases. Of those, 42 patients $(17.1 \%)$ underwent ITAI during their procedure. Patients who underwent ITAI for tumor debulking were generally middle-aged (65.5, IQR 57.872 years), predominantly men $(64.3 \%)$ and of Caucasian ancestry (95\%). The most common histopathology of the debulked tumors was squamous cell carcinoma (52.4\%) followed by adenocarcinoma (21.4\%), metastatic cancer $(14.3 \%)$, small cell carcinoma $(7.1 \%)$ and carcinoid tumor $(4.8 \%)$. More than half of the patients had stage IV malignancy at the time of the bronchoscopy (57.1\%). Most of the patients had ECOG performance status score of either ECOG 2 (40.5\%) or ECOG 3 (38.1\%). The obstruction was most commonly located in the left main stem $(38.1 \%)$ followed by right main stem/bronchus intermedius (35.7\%) (Table 1).

\section{Procedure characteristics}

Sixty-nine percent of the patients underwent two bronchoscopies and $12 \%$ had three bronchoscopies. The median duration of the initial debulking bronchoscopy was 37 minutes (IQR 28-48 minutes) and the subsequent procedure durations were 31 minutes (IQR 25-38.3 minutes). The median duration between the first and last procedure was 4 days (IQR 3-6 days). Around $90 \%$ of the patients underwent APC with mechanical debulking; six patients $(14 \%)$ had a stent placed and one patient $(2.4 \%)$ had balloon dilatation. The procedures were generally welltolerated and only one patient had significant bleeding that required fluid resuscitation and intensive care unit monitoring for 24 hours after the procedure. This bleeding episode was in the subsequent bronchoscopy and ITAI 
Table 1 Baseline demographics and procedural characteristics of patients with obstructing endobronchial tumors who underwent ITAI

\begin{tabular}{|c|c|}
\hline Variable & Value \\
\hline Total number of patients & 42 \\
\hline Age, years [IQ range] & $65.5[57.8-72]$ \\
\hline Women (\%) & $15(35.7)$ \\
\hline White (\%) & $40(95.0)$ \\
\hline \multicolumn{2}{|l|}{ Histopathology (\%) } \\
\hline Squamous cell carcinoma & $22(52.4)$ \\
\hline Adenocarcinoma & $9(21.4)$ \\
\hline Metastatic cancer & $6(14.3)$ \\
\hline Small cell carcinoma & $3(7.1)$ \\
\hline Carcinoid & $2(4.8)$ \\
\hline \multicolumn{2}{|l|}{ Cancer stage } \\
\hline Stage 1 & $2(4.8)$ \\
\hline Stage 2 & $2(4.8)$ \\
\hline Stage 3 & $13(28.6)$ \\
\hline Stage 4 & $24(57.1)$ \\
\hline Not available & $2(4.8)$ \\
\hline \multicolumn{2}{|l|}{ ECOG performance status (\%) } \\
\hline ECOG 1 & $4(9.5)$ \\
\hline ECOG 2 & $17(40.5)$ \\
\hline ECOG 3 & $16(38.1)$ \\
\hline ECOG 4 & $3(7.1)$ \\
\hline Not available & $2(4.8)$ \\
\hline \multicolumn{2}{|l|}{ Location (\%) } \\
\hline Left main stem & $16(38.1)$ \\
\hline Right main stem/bronchus intermedius & $15(35.7)$ \\
\hline Left upper lobe & $6(14.2)$ \\
\hline Right middle lobe & $2(4.8)$ \\
\hline Trachea & $1(2.4)$ \\
\hline Left lower lobe & $1(2.4)$ \\
\hline Right lower lobe & $1(2.4)$ \\
\hline Duration of initial procedure, minutes [IQ range] & 37 [28-48] \\
\hline $\begin{array}{l}\text { Duration of subsequent procedures, minutes [IQ } \\
\text { range] }\end{array}$ & $31[25-38.3]$ \\
\hline Days between first and last procedure [IQ range] & $4[3-6]$ \\
\hline
\end{tabular}

Table 1 (continued)
Table 1 (continued)

\begin{tabular}{|c|c|}
\hline Variable & Value \\
\hline Subsequent injections in repeat procedures (\%) & $7(16.7)$ \\
\hline \multicolumn{2}{|l|}{$\begin{array}{l}\text { Additional bronchoscopic therapeutic interventions } \\
\text { (\%) }\end{array}$} \\
\hline APC (including corecath) & $38(90.5)$ \\
\hline Mechanical debulking (including snare) & $37(88.1)$ \\
\hline Stent placement & $6(14.3)$ \\
\hline Balloon dilation & $1(2.4)$ \\
\hline Cisplatin injection & $3(7.2)$ \\
\hline None & $1(3.0)$ \\
\hline \multicolumn{2}{|l|}{ Complications in any procedure (\%) } \\
\hline $\begin{array}{l}\text { Mild bleeding (spontaneous resolution without } \\
\text { intervention) }\end{array}$ & $2(4.8)$ \\
\hline Severe bleeding (required fluid resuscitation) & $1(2.4)$ \\
\hline \multicolumn{2}{|l|}{$\begin{array}{l}\text { Airway obstruction response to } \\
\text { interventions }(\%)^{\dagger}\end{array}$} \\
\hline Good response ( $\geq 50 \%$ response) & $29(83.0)$ \\
\hline Low response (<50\% response) & $6(17.0)$ \\
\hline 6-month mortality (\%) & $21(55.3)$ \\
\hline
\end{tabular}

was not used in that procedure. No other complications were reported. Twenty-one patients $(55.3 \%)$ were dead at 6-month follow up (Table 1).

\section{Treatment response}

Alcohol injection made it easier to debulk the tumor on subsequent procedures as subjectively measured by the interventional pulmonologists performing the procedure. Of the patients that underwent repeat bronchoscopy to evaluate airway patency response (34 patients), 28 patients (82\%) had a good airway patency response ( $\geq 50 \%$ response) at the conclusion of the last procedure. Of the six patients classified to have a low response, three patients had airway patency response between $25-50 \%$. Eight patients underwent ITAI in the first bronchoscopy but did not have a repeat procedure to evaluate response as the patient and/ or family opted to pursue comfort measures and not to have 
any further interventions.

Patients with good response were more likely to be of Caucasian ancestry when compared to those with low response $(100 \%$ vs. $67 \%, \mathrm{P}=0.027)$. There were no other differences in demographics between the two groups. Additionally, patients with good response were less likely to have lung adenocarcinoma on histopathologic examination ( $18 \%$ vs. $67 \%, \mathrm{P}=0.031$ ) with no other differences noted in histopathology, cancer staging, ECOG performance score, additional bronchoscopic interventions, or concurrent systemic therapies administered (Table 2).

\section{Selected cases}

\section{Patient 1}

A 50-year-old woman was diagnosed with advanced right upper lobe adenocarcinoma. She underwent concurrent chemoradiation. Unfortunately, she progressed and presented with cough, hemoptysis, and worsening dyspnea. Initial bronchoscopy (Figure 1A) showed complete right main bronchus obstruction. Alcohol was injected at the end of the first intervention. Repeat bronchoscopy two days later showed significant tissue necrosis with pseudomembrane formation (Figure 1B). Tumor debulking was performed and airway patency was successfully achieved at the end of the second procedure (Figure 1C) with no complications.

\section{Patient 2}

A 66-year-old man presented with cough, sputum, hemoptysis and post-obstructive pneumonia. Initial bronchoscopy (Figure 2A) showed complete right main stem bronchus obstruction secondary to squamous cell carcinoma. ITAI was performed at the conclusion of the first procedure. Repeat bronchoscopy two days later again showed pseudo-membrane formation with tissue necrosis (Figure 2B). He successfully underwent tumor debulking with airway stent placement (Figure 2C).

\section{Discussion}

In this report, we demonstrated that endobronchial ITAI can be used as an adjunct debulking modality to help relieve malignant central airway obstruction. We managed to successfully achieve airway patency in the majority of our patients. Endobronchial ITAI was used when conventional debulking methods were deemed insufficient to attain good results and significant airway obstruction remained following the usual bronchoscopic techniques to achieve airway patency. ITAI had a good safety profile with no major complications reported.

Different bronchoscopic debulking modalities can be used to achieve airway patency. These include mechanical debulking and heat modalities such as neodymium-doped yttrium aluminum garnet (YAG) laser, APC, electrocautery and cryosurgery. Photodynamic therapy (PDT) can also be used. The choice of therapy is typically dictated by the patient's stability, tumor characteristics, technology availability, clinical expertise, and physician preference (16). Sometimes, a large tumor burden and bleeding tendency makes it difficult to debulk the tumor and relieve the obstruction despite exhaustive multimodality approaches. Also, some of these patients with central airway obstruction may not be able to tolerate a prolonged procedure due to underlying co-morbidities.

The use of alcohol injection was first introduced in 1983 in the context of HCC by Ebara et al. and has been used since then with a good safety profile over the years (17). Pure alcohol, when injected directly in the tumor, can induce tumor cell necrosis (9). Furthermore, alcohol can cause microcirculatory embolization inducing direct protein denaturing, platelet aggregation, and vascular endothelial cells dehydration which can inhibit the tumor growth and decrease the blood supply to the injected area $(18,19)$. Trans-arterial alcohol injection has also been described for the treatment of HCC causing complete infarction of the tumor tissue with good results (9). Additionally, pure alcohol is a well-known sclerosing agent causing complete blood vessel thrombosis when injected to treat vascular malformations, esophageal varices and vascular renal tumors $(10,11)$. Our idea of visually injecting dehydrated alcohol in endobronchial tumors was inspired by these reports and we were able to directly visualize the tissue necrosis induced by alcohol injection.

In our experience, we have opted to use ITAI in difficult cases with large tumor burden to ease the debulking process, during debulking cases which lead to bleeding significant enough to obscure the bronchoscopic image requiring repeated cleaning, and insufficient airway patency following the usual airway interventions. We typically inject the alcohol at the end of the first debulking procedure. In subsequent procedures, we notice significant tumor necrosis and pseudo-membrane formation, which makes it easier to excise the tumor and achieve airway patency. Much of the necrotic tissue can be removed via regular suctioning and use of forceps. Additionally, we have noticed 
Table 2 Response to endobronchial intra-tumor alcohol injection based on demographics, tumor type, concurrent systemic therapy, procedural features and additional airway maneuvers ${ }^{* \dagger}$

\begin{tabular}{|c|c|c|c|}
\hline Variable & Good response $(n=28)$ & Low response $(n=6)$ & $P$ value \\
\hline Age, years [IQ] & 64 [56-73] & 67 [61-72] & 0.382 \\
\hline Women, n (\%) & $12(42.9)$ & $1(16.7)$ & 0.231 \\
\hline \multicolumn{4}{|l|}{ Race } \\
\hline White, n (\%) & $28(100)$ & $4(66.7)$ & \\
\hline \multicolumn{4}{|l|}{ Histopathology, n (\%) } \\
\hline Squamous cell carcinoma & $13(46.4)$ & $2(33.3)$ & 0.452 \\
\hline Adenocarcinoma & 5 (17.9) & $4(66.7)$ & 0.031 \\
\hline Metastatic cancer & $5(17.9)$ & 0 & 0.353 \\
\hline \multicolumn{4}{|l|}{ Stage ${ }^{\S}$} \\
\hline Stage 1 & $2(7.7)$ & 0 & 0.655 \\
\hline Stage 2 & $2(7.7)$ & 0 & 0.655 \\
\hline Stage 3 & $5(19.2)$ & $3(50.0)$ & 0.148 \\
\hline Stage 4 & $17(65.4)$ & $3(50.0)$ & 0.399 \\
\hline \multicolumn{4}{|l|}{ ECOG performance status } \\
\hline 1 & $4(14.8)$ & 0 & \\
\hline 2 & $13(48.1)$ & $3(60.0)$ & 0.777 \\
\hline Mechanical debulking & $25(89.3)$ & 5 (83.3) & 0.559 \\
\hline Stent placement & $5(17.9)$ & 0 & 0.353 \\
\hline Cisplatin injection & $3(10.7)$ & 0 & 0.547 \\
\hline Balloon dilation & $1(3.6)$ & 0 & 0.824 \\
\hline \multicolumn{4}{|l|}{ Concurrent systemic therapy" } \\
\hline Chemotherapy & $18(69.2)$ & $4(80.0)$ & 0.542 \\
\hline Immune therapy & $6(23.1)$ & $2(40.0)$ & 0.385 \\
\hline Radiation therapy & $13(50.0)$ & $2(40.0)$ & 0.532 \\
\hline None & $5(19.2)$ & $1(20.0)$ & 0.687 \\
\hline 6-month mortality (\%) & $9(32.1)$ & 5 (83.3) & 0.021 \\
\hline
\end{tabular}

*, good response defined if $\geq 50 \%$ airway patency restoration was achieved, low response if $<50 \%$ airway patency was achieved; ${ }^{\dagger}$, medians and IQ ranges are presented for continuous variables; ${ }^{\S}$, data regarding staging was missing for two patients from the good response group; ", data regarding concurrent systemic therapy was missing for two patients from the good response group and one patient from the low response group. APC, argon plasma coagulation; ECOG, Eastern Cooperative Oncology Group; IQ, interquartile. 

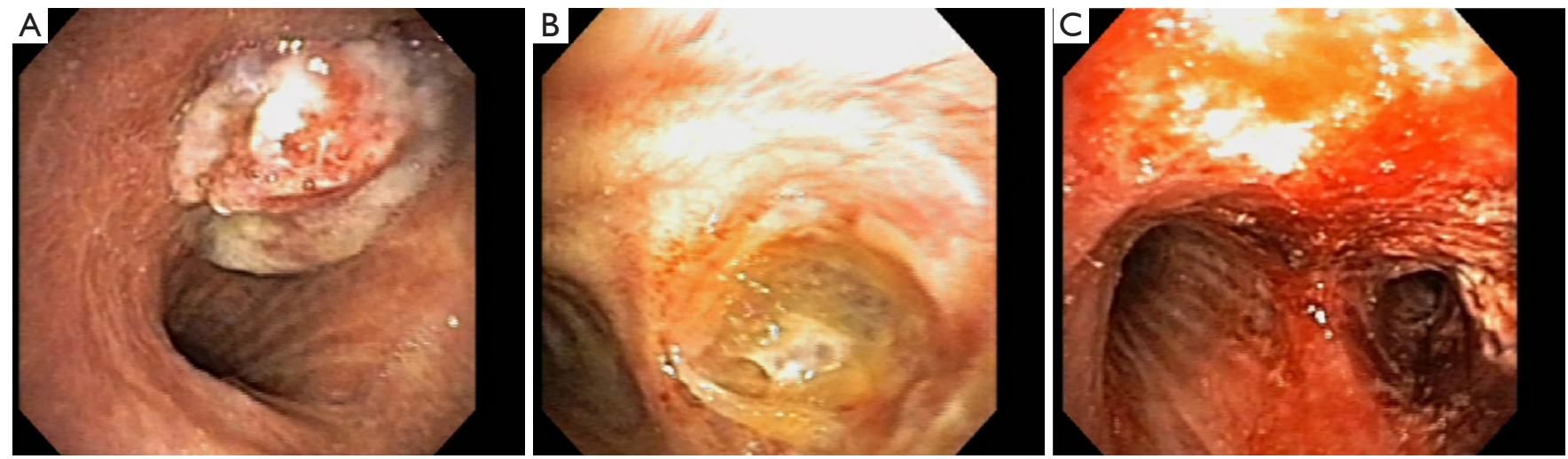

Figure 1 Bronchoscopy images of a 50-year-old woman with malignant complete right bronchus obstruction before and after tumor debulking while using alcohol injection. (A) Initial bronchoscopic image of a 50-year-old woman with lung adenocarcinoma showing complete right bronchus obstruction; (B) tissue necrosis and pseudo-membrane formation noted on repeat bronchoscopy two days after intra-tumor-alcohol-injection; (C) airway patency was successfully achieved at the conclusion of the second debulking bronchoscopy.
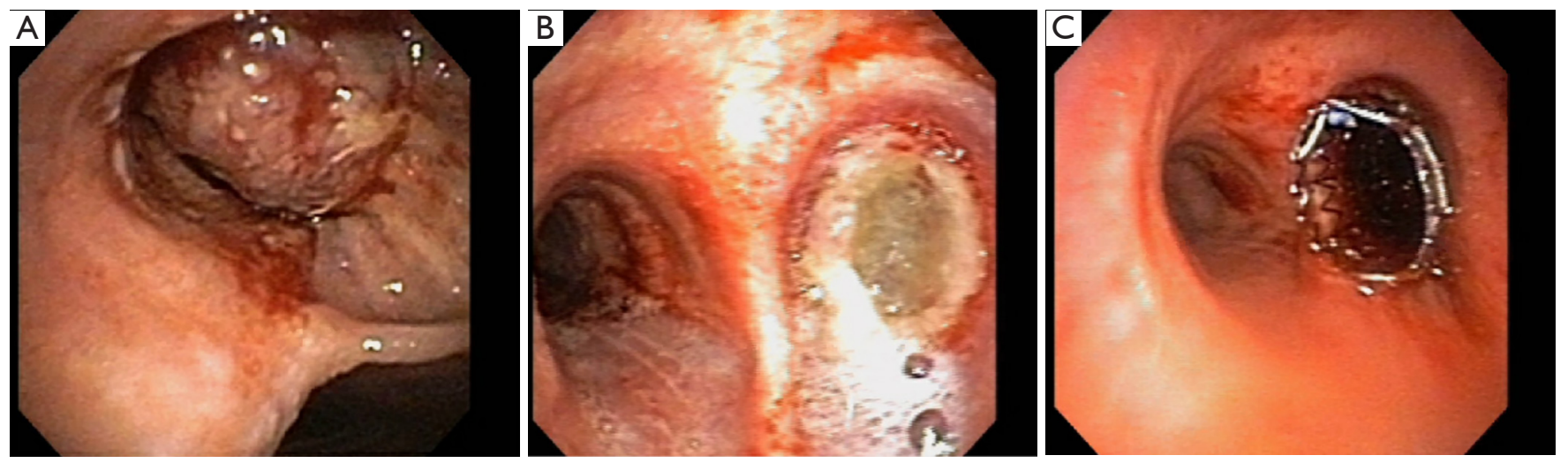

Figure 2 Bronchoscopy images of a 66-year-old man with malignant complete right main stem occlusion before and after tumor debulking using alcohol injection and airway stent placement. (A) Initial bronchoscopic image of a 66-year-old man with lung squamous cell carcinoma showing complete right main stem occlusion; (B) bronchoscopic view of the tumor two days after intra-tumor-alcohol-injection showing significant necrosis and pseudo-membrane formation; (C) bronchoscopic picture showing successful achievement of airway patency and airway stent placement.

decreased bleeding tendency after ITAI. Furthermore, ITAI is inexpensive and does not significantly increase the length of the procedure and can potentially decrease the overall duration of the staged subsequent procedure. However, in our cohort, the airway patency achieved cannot be solely attributed to ITAI alone as it was used as part of a multimodality approach and, as such, is a limitation to our study. Achieving airway patency has been shown in previous studies to be associated with better survival and quality of life $(6,20,21)$. This survival and quality of life benefit reported in previous studies from achieving airway patency might be attributed to the potential clinical improvement that would act as a bridge to allow receiving more systemic therapies sessions such as chemotherapy or radiation therapy (22). Additionally, relieving the obstruction might help the treatment of any post-obstructive pneumoniae and resulting sepsis.

To our knowledge, this is the first report to describe the use of dehydrated alcohol injection in debulking of central airway tumors. This report provides a descriptive data of a novel procedure that can be potentially used in difficult airway obstruction cases. In our experience as mentioned 
above, one of the major advantages of using endobronchial ITAI is the ability to achieve significant airway patency, which probably could not have been achieved using conventional tumor ablation and debulking modalities due to tumor bulk, extent of mucosal infiltration, or bleeding of the tumor. We performed endobronchial ITAI only in those patients where all conventional options including but not limited to ablative therapies, debulking, and stenting modalities were exhausted or thought to be not feasible. In some patients $(n=5)$, endobronchial stent placement was achieved at the conclusion of debulking procedures with the aid of ITAI. Another advantage of endobronchial ITAI includes decreased procedural duration on subsequent procedures as it helps with debulking and bleeding. ITAI, however, does require follow-up bronchoscopy to achieve maximal airway patency. Potential side effects from ITAI might include airway fire, airway perforation or fistula formation if appropriate measures are not taken especially if larger doses were used or if used in combination with heat (alcohol therapy followed by APC or electrocautery). In our study, ITAI was well-tolerated with minimal adverse events.

We acknowledge several limitations. First, our report was a retrospective chart review study, which can be limited by the adequacy of documentation in medical records. Unfortunately, data regarding the post procedural performance status and quality of life was lacking. Second, this is a single center study with a relatively small sample size; hence, the generalizability of our results may be limited. Third, it is difficult to quantify the exact impact of ITAI alone on relieving airway obstruction as it was used in a multimodality approach. It also adds an additional procedure to fragile patients with advanced cancers. However, at the discretion of the bronchoscopist in our cohort, many of these patients would have been left with significant airway obstruction if we only used conventional modalities. It would be ideal to perform a randomized trial of bronchoscopic intervention with and without ITAI, however, randomized trials in these patient populations are hard to perform. We acknowledge that our division of quantifying airway obstruction method, by visual inspection is inconsistent with the potential for error both in over and underestimating and lacks any type of uniform standard. Tissue necrosis following ITAI cannot be fully attributed to alcohol as other ablative modalities can cause similar changes.

Further studies are warranted to determine whether ITAI is superior to other interventional methods, especially regarding duration of response, as well as to standardize the treatment protocol and determine the effects of ITAI on patient symptoms and quality of life. Intra-tumoral alcohol may possibly be able to be used to treat peripheral tumors, potentially in combination with stereotactic radiation. This application should also be studied in future trials.

\section{Conclusions}

Dehydrated alcohol is readily available, inexpensive and a safe drug that can be potentially added to the interventional pulmonologists' armamentarium. ITAI can induce tumor necrosis and pseudo-membrane formation that can ease the tumor debulking process. It has a good safety profile and can be used in a multimodality approach to achieve airway patency when traditional modalities fail or reach their limitation.

\section{Acknowledgments}

Funding: None.

\section{Footnote}

Reporting Checklist: The authors have completed the STROBE reporting checklist. Available at https://dx.doi. org/10.21037/jtd-20-3554

Data Sharing Statement: Available at https://dx.doi. org/10.21037/jtd-20-3554

Conflicts of Interest: All authors have completed the ICMJE uniform disclosure form (available at https://dx.doi. org/10.21037/jtd-20-3554). The authors have no conflicts of interest to declare.

Ethical Statement: The authors are accountable for all aspects of the work in ensuring that questions related to the accuracy or integrity of any part of the work are appropriately investigated and resolved. The study was conducted in accordance with the Declaration of Helsinki (as revised in 2013). The study was approved by UF Institutional Review Board (protocol no. IRB202001086). All patients signed informed consent before the procedure.

Open Access Statement: This is an Open Access article distributed in accordance with the Creative Commons Attribution-NonCommercial-NoDerivs 4.0 International License (CC BY-NC-ND 4.0), which permits the non- 
commercial replication and distribution of the article with the strict proviso that no changes or edits are made and the original work is properly cited (including links to both the formal publication through the relevant DOI and the license). See: https://creativecommons.org/licenses/by-nc-nd/4.0/.

\section{References}

1. Ernst A, Feller-Kopman D, Becker HD, et al. Central airway obstruction. Am J Respir Crit Care Med 2004;169:1278-97.

2. Kuo SC, Lo YL, Chou CL, et al. Bronchoscopic debulking for endobronchial malignancy: Predictors of recanalization and recurrence. Thorac Cancer 2015;6:722-30.

3. Roswit B, Patno ME, Rapp R, et al. The survival of patients with inoperable lung cancer: a large-scale randomized study of radiation therapy versus placebo. Radiology 1968;90:688-97.

4. Chetty KG, Moran EM, Sassoon CS, et al. Effect of radiation therapy on bronchial obstruction due to bronchogenic carcinoma. Chest 1989;95:582-4.

5. Hazuka MB, Bunn PA Jr. Controversies in the nonsurgical treatment of stage III non-small cell lung cancer. Am Rev Respir Dis 1992;145:967-77.

6. Mehta HJ, Begnaud A, Penley AM, et al. Restoration of Patency to Central Airways Occluded by Malignant Endobronchial Tumors Using Intratumoral Injection of Cisplatin. Ann Am Thorac Soc 2015;12:1345-50.

7. Freitag L, Ernst A, Thomas M, et al. Sequential photodynamic therapy (PDT) and high dose brachytherapy for endobronchial tumour control in patients with limited bronchogenic carcinoma. Thorax 2004;59:790-3.

8. Matsui O, Kadoya M, Yoshikawa J, et al. Small hepatocellular carcinoma: treatment with subsegmental transcatheter arterial embolization. Radiology 1993;188:79-83.

9. Gu YK, Luo RG, Huang JH, et al. Transarterial embolization ablation of hepatocellular carcinoma with a lipiodol-ethanol mixture. World J Gastroenterol 2010;16:5766-72.

10. Ellman BA, Parkhill BJ, Curry TS 3rd, et al. Ablation of renal tumors with absolute ethanol: a new technique. Radiology 1981;141:619-26.

11. Yakes WF, Haas DK, Parker SH, et al. Symptomatic vascular malformations: ethanol embolotherapy. Radiology 1989;170:1059-66.

12. Livraghi T, Festi D, Monti F, et al. US-guided percutaneous alcohol injection of small hepatic and abdominal tumors. Radiology 1986;161:309-12.

13. Gobin YP, Murayama Y, Milanese K, et al. Head and neck hypervascular lesions: embolization with ethylene vinyl alcohol copolymer--laboratory evaluation in Swine and clinical evaluation in humans. Radiology 2001;221:309-17.

14. Wang G, Li J, Zhang H, et al. Anhydrous ethanol reduces intraoperative hemorrhage of intracranial highly vascularized large meningiomas. Chin. -Ger. J. Clin. Oncol 2010;9:344-8.

15. Crescenzi A, Papini E, Pacella CM, et al. Morphological changes in a hyperfunctioning thyroid adenoma after percutaneous ethanol injection: histological, enzymatic and sub-microscopical alterations. J Endocrinol Invest 1996;19:371-6.

16. Criner GJ, Eberhardt R, Fernandez-Bussy S, et al. Interventional Bronchoscopy. Am J Respir Crit Care Med 2020;202:29-50.

17. Ebara M, Okabe S, Kita K, et al. Percutaneous ethanol injection for small hepatocellular carcinoma: therapeutic efficacy based on 20-year observation. J Hepatol 2005;43:458-64.

18. Kan Z, Wallace $S$. Transcatheter liver lobar ablation: an experimental trial in an animal model. Eur Radiol 1997;7:1071-5.

19. Lin XD, Lin LW. Local injection therapy for hepatocellular carcinoma. Hepatobiliary Pancreat Dis Int 2006;5:16-21.

20. Stratakos G, Gerovasili V, Dimitropoulos C, et al. Survival and Quality of Life Benefit after Endoscopic Management of Malignant Central Airway Obstruction. J Cancer 2016;7:794-802.

21. Razi SS, Lebovics RS, Schwartz G, et al. Timely airway stenting improves survival in patients with malignant central airway obstruction. Ann Thorac Surg 2010;90:1088-93.

22. Kim JH, Shin JH, Song HY, et al. Palliative treatment of inoperable malignant tracheobronchial obstruction: temporary stenting combined with radiation therapy and/or chemotherapy. AJR Am J Roentgenol 2009;193:W38-42.

Cite this article as: Jantz MA, Omballi M, Alzghoul BN, Fernandez Bussy S, Becnel D, Majid A, Mehta HJ. Utility of bronchoscopic intra-tumoral alcohol injection to restore airway patency. J Thorac Dis 2021;13(8):4956-4964. doi: 10.21037/jtd-20-3554 